EDITORIAL

\title{
Atrial fibrillation: an emerging epidemic?
}

\author{
J S Steinberg
}

An aging general population, coupled with improved treatments for those individuals with coronary artery disease, hypertension, or heart failure, has led to a dramatic increase in the prevalence of atrial fibrillation

Heart 2004;90:239-240. doi: 10.1136/hrt.2003.014720

care, treatment complications, and the consequences of additional intervention and length of stay when AF complicates another illness, would increase the financial costs of this disease greatly. There is no reason to believe that similar economic consequences are not being experienced in other developed societies, including the USA. trial fibrillation $(\mathrm{AF})$ is the most common
arrhythmic condition facing physicians, afflicting almost 1 in 20 people over the age of $60 .{ }^{1}$ The prevalence of $\mathrm{AF}$ is on the rise, due, in part, to an aging general population and to increased longevity resulting from improved medical care among patients with coronary artery disease, hypertension and heart failureall chronic cardiac conditions which predispose to $\mathrm{AF}^{2}{ }^{2}$ It is estimated that slightly less than $1 \%$ of the general population (well over two million in the USA) currently have AF, and the prevalence will increase 2.5 -fold by the year 2050. ${ }^{1}$ Prevalence figures for AF are underestimates because AF may be undiagnosed if sporadic and if not associated with significant symptoms.

AF is clinically important because it contributes to the incidence of heart failure, stroke, and both overall and cardiovascular mortality. The mechanism(s) by which AF contributes to increased mortality rate r-6 $^{4-6}$ is currently uncertain. Moreover, no treatment of AF to date has resulted in a lower overall death rate. ${ }^{7}$ Indeed, it is conceivable that conventional treatment with antiarrhythmic drugs may contribute to the higher death rate observed in AF populations. ${ }^{78}$ Equally troubling are the potential financial burdens associated with this chronic and increasingly common condition. Since we can only expect continued trends in both the aging of the general population and further successful efforts at staving off death from chronic cardiovascular disease, formal study of the financial considerations posed by atrial fibrillation is quite timely.

\section{FINANCIAL COST OF ATRIAL FIBRILLATION}

The report in this issue of Heart by Stewart and colleagues ${ }^{9}$ provides a thorough analysis of the costs associated with the care of patients with AF in the UK. These data indicate that the costs of AF care, already substantial, are rising dramatically. At present, the authors estimate that in the

Correspondence to: Jonathan S Steinberg, MD, St Luke's-Roosevelt Hospital Center, 1111 Amsterdam Avenue, New York, NY 10025, USA; jss7@columbia.edu
What should the reaction be to the compelling results gathered by Stewart and colleagues? ${ }^{9}$ Specifically, what are the resources that could be marshalled to combat the AF epidemic and/or its economic costs? The most desired strategythe prevention of $\mathrm{AF}$-is currently thwarted by the pattern of AF incidence. While multifactorial in origin, the disease is frequently tightly coupled to the aging process of the atria and related cardiac structures. But intriguing new concepts suggest that AF may also occur as a consequence of ventricular diastolic dysfunction, ${ }^{3}$ which is in turn a product of an aging ventricle. This suggests a compulsory therapeutic intervention: aggressive treatment of hypertension. Second, genetic predisposition, ${ }^{10}$ possibly involving a variety of electrophysiologic and/or facilitating factors, represents a potential venue of future therapeutic investigation. Third, direct curative intervention by catheter techniques has the potential to reduce AF prevalence swiftly. Other supraventricular tachyarrhythmias (SVT) are regularly cured by catheter ablation, virtually eliminating chronic care for these arrhythmic conditions.

\section{ABLATION OF TRIGGER SITES}

Although not yet at the success rates for SVT, important progress has been made for AF. Ablation of the most common trigger sites of AF (the pulmonary veins) has yielded impressive response rates, as high as approximately $70-75 \%$, although the atrial substrate remains untreated. This approach can result in cure in a subset of patients whose AF is primarily related to an abnormal trigger focus, ${ }^{11}$ but many others may ultimately require a more extensive catheter approach. Targeting of the atrial myocardium, especially the posterior left atrium, ${ }^{12}$ along with pulmonary vein ablation, is a promising new development that may herald a new interventional era of effective permanent alteration of atrial substrate. However, there are no long term studies on this approach yet. If preliminary findings continue to be favourable, a large scale randomised controlled clinical trial to test the value of interventional catheter therapy of most forms of $\mathrm{AF}$ relative to conventional medical treatment would then be warranted.

Stewart and colleagues emphasise another powerful driver of the cost of AF care: an
Further, the current study indicates that these figures are probably an underestimation of expenditures. Factoring in the costs of stroke
UK about $1 \%$ of all National Health Service expenditures are the result of AF. These costs have doubled in only a five year time span. 
increasing frequency of hospitalisation. ${ }^{9}$ About 1 in 10 patients with diagnosed AF in this study were hospitalised because of a primary diagnosis suggesting AF. No data were available to explain this phenomenon, but inpatient care may be required to treat elderly or sicker patients who experience $\mathrm{AF}$, to initiate and assess drug treatment, and to treat the complications of AF, particularly stroke. It is also apparent in clinical practice that many patients with AF are hospitalised for no specific reason other than the presence of AF, often transient and idiopathic, or for simple therapeutic interventions. Some of these admissions may be unnecessary. Accordingly, specific criteria for hospitalisation should be developed that would aid emergency department, primary care, and cardiology physicians with the optimal use of expensive resources (the hospital) versus safe and effective outpatient alternatives. A major reduction in admission to, and stay in, the hospital would also result in major cost savings.

\section{IMPACT OF STROKE}

Finally, Stewart and colleagues emphasise the impact of stroke and all its clinical ramifications on the cost of AF care. ${ }^{9}$ In this regard, effective preventive treatment is already available but underutilised. Warfarin has been proven to reduce stroke incidence by about two thirds in high risk AF patients, yet surveys repeatedly indicate that many patients who should be anticoagulated are not. ${ }^{13-15}$ There is no disputing that chronic treatment with warfarin is a practical challenge, but clearly a much higher proportion of eligible patients should be treated. The introduction of direct thrombin inhibitors, recently shown to be equivalent to warfarin $^{16}$ and which do not require blood testing for assessing therapeutic effect, should herald an era of much greater use of chronic anticoagulation treatment in patients with AF at risk of stroke. The consequence of this effort will likely be fewer strokes and thus less health care expenditures.

In summary, Stewart and colleagues demonstrate that AF represents a costly threat to the financial stability of health care systems. As the economic burdens of this illness continue to rise, it behoves the medical community to avail itself of the formidable approaches that are becoming available to prevent and modify this chronic illness, and for funding agencies to increase resources that promote research into the treatment and prevention of $\mathrm{AF}$.

\section{REFERENCES}

1 Go AS, Hylek EM, Phillips KA, et al. Prevalence of diagnosed atrial fibrillation in adults. JAMA 2001;285:2370-5.

2 Wolf PAS, Mitchell JB, Baker CS, et al. Impact of atrial fibrillation on mortality, stroke, and medical costs. Arch Intern Med 1998;158:229-34.

3 Tsang TSM, Gersh BJ. Atrial fibrillation: an old disease, a new epidemic. Am J Med 2002; 1 13:432-5

4 Benjamin EJ, Wolf PA, D'Agostino RB, et al. Impact of atrial fibrillation on the risk of death: the Framingham Heart Study. Circulation 1998;98:946-52.

5 Vidaillet H, Granada JF, Chyou PH, et al. A population-based study of mortality among patients with atrial fibrillation or flutter. Am J Med 2002;113:365-70.

6 Stewart S, Hart CL, Hole DJ, et al. A population-based study of the long-term risks associated with atrial fibrillation: 20-year follow-up of the Renfrew/ Paisley study. Am J Med 2002;113:359-64

7 The Atrial Fibrillation Follow-up Investigation of Rhythm Management (AFFIRM) Investigators. A comparison of rate control and rhythm control in patients with atrial fibrillation. N Engl J Med 2002;347:1825-33.

8 Steinberg JS, Sadaniantz A, Miller JM, et al. Comparison of modes of death between randomized treatment strategies of the atrial fibrillation follow-up investigation of rhythm management (AFFIRM) study. Circulation (in press)

9 Stewart S, Murphy N, Walker A, et al. Cost of an emerging epidemic: an economic analysis of atrial fibrillation in the UK. Heart 2004;90:286-92.

10 Brugada R, Tapscott T, Czernuszewicz $G$, et al. Identification of a genetic locus for familial atrial fibrillation. N Engl J Med 1997;336:905-1 1.

11 Haissaguerre $M$, Jais $P$, Shah DC, et al. Spontaneous initiation of atrial fibrillation by ectopic beats originating in the pulmonary veins. N Engl J Med 1998;339:659-66.

12 Jais P, Weerasooriya R, Scavee C, et al. Catheter ablation of chronic atrial fibrillation using a combination of left linear lesions and pulmonary vein isolation [abstract]. PACE 2003;26:962.

13 Whittle J, Whickenheiser L, Venditti LN. Is warfarin underused in the treatment of elderly persons with atrial fibrillation? Arch Intern Med 1997; 1 197:441-5.

14 Brass LM, Krumholz JM, Scinto JM, et al. Warfarin use among patients with atrial fibrillation. Stroke 1997;28:2382-9.

15 Albers GW, Yim JM, Bittar N, et al. Status of antithrombotic therapy for patients with atrial fibrillation in university hospitals. Arch Intern Med 1996; 156:2311-6

16 The Executive Steering Committee on behalf of the SPORTIF III Investigators. Stroke prevention using the oral direct thrombin inhibitor ximelagatran in patients with nonvalvular atrial fibrillation (SPORTIF), Presented at American College of Cardiology Annual Scientific Sessions 2003

\section{ELECTRONIC PAGES}

\section{Heart Online case reports: www.heartjnl.com}

$\mathrm{T}$

he follow electronic only articles are published in conjunction with this issue of Heart.

\section{Congenital coronary artery anomaly simulating an acute aortic dissection}

\section{De Luca, F Bovenzi, I de Luca}

In this rare case, a patient had an anomalous right coronary artery originating from the left sinus of Valsalva coursing between the great vessels. He presented with symptoms and computed tomograms suggestive of an acute aortic dissection.

(Heart 2004;90:el1) www.heartjnl.com/cgi/content/full/90/ $3 / \mathrm{ell}$

\section{Acute myocardial infarction probably caused by paradoxical embolus in a pregnant woman \\ P Agostoni, G Gasparini, G Destro}

A 19 year old pregnant woman presented to the coronary care unit with an acute anterior myocardial infarction. She was treated with primary percutaneous transluminal coronary angioplasty of the proximal left anterior descending coronary artery. Ultrasound examination showed patent foramen ovale (PFO) and atrial septal aneurysm. The patient was a heterozygote carrier of factor V Leiden. Despite the lack of a clear clue, it was considered that the pathophysiological cause of this infarction was a paradoxical embolus in the left coronary artery. Pregnancy and factor V Leiden carriership are associated with increased risk of venous thromboembolism and the association between PFO and atrial septal aneurysm is a strong risk factor for systemic embolisation.

(Heart 2004;90:el2) www.heartjnl.com/cgi/content/full/90/ 3/e12 